\title{
Identification of the soluble form of tyrosine kinase receptor Axl as a potential biomarker for intracranial aneurysm rupture
}

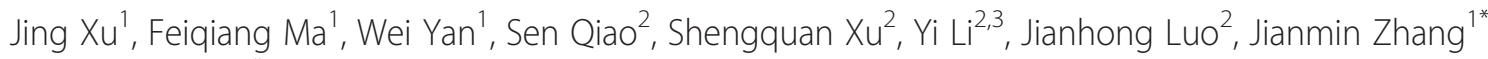
and Jinghua $\operatorname{~in}^{2^{*}}$

\begin{abstract}
Background: Subarachnoid hemorrhage caused by a ruptured intracranial aneurysm (RIA) is a devastating condition with significant morbidity and mortality. Despite the fact that RIAs can be prevented by microsurgical clipping or endovascular coiling, there are no reliable means of effectively predicting IA patients at risk for rupture. The purpose of our study was to discover differentially-expressed glycoproteins in IAs with or without rupture as potential biomarkers to predict rupture.

Methods: Forty age/gender-matched patients with RIA, unruptured IA (UIA), healthy controls (HCs) and disease controls (DCs) (discovery cohort, $\mathrm{n}=10$ per group) were recruited and a multiplex quantitative proteomic method, iTRAQ (isobaric Tagging for Relative and Absolute protein Quantification), was used to quantify relative changes in the lectin-purified glycoproteins in CSF from RIAs and UIAs compared to HCs and DCs. Then we verified the proteomic results in an independent set of samples (validation cohort, $n=20$ per group) by enzyme-linked immunosorbent assay. Finally, we evaluated the specificity and sensitivity of the candidate marker with receiver operating characteristic (ROC) curve methods.

Results: The proteomic findings identified 294 proteins, 40 of which displayed quantitative changes unique to RIA, 13 to UIA, and 20 to IA. One of these proteins, receptor tyrosine kinase Axl, was significantly increased in RIA, as confirmed in CSF from the discovery cohort as well as in CSF and plasma from the validation cohort $(p<0.05)$. Spearman's correlation analysis revealed that the CSF and plasma Axl levels were strongly correlated $(r=0.93$, $p$ <0.0001). The ROC curve indicated an optimal CSF Axl threshold of $0.12 \mathrm{nM}$ for discriminating RIA from UIA with corresponding sensitivity/specificity of $73.33 \% / 90 \%$ and an area under the curve (AUC) of 0.89 (95\% Cl: 0.80-0.97, $p<0.0001$ ). The optimal threshold for plasma Axl was $1.7 \mathrm{nM}$ with corresponding sensitivity/ specificity of $50 \% / 80 \%$ and an AUC of 0.71 (95\% Cl: $0.54-0.87, p=0.027$ ).
\end{abstract}

Conclusions: Both CSF and plasma Axl levels are significantly elevated in RIA patients. Axl might serve as a promising biomarker to predict the rupture of IA.

Keywords: Intracranial aneurysm, Rupture, Cerebrospinal fluid, Proteomics, Biomarker, Glycoproteins

\footnotetext{
*Correspondence: zjm135@vip.sina.com; jhjin@zju.edu.cn

'Department of Neurosurgery, The Second Affiliated Hospital of Zhejiang

University School of Medicine, Hangzhou, Zhejiang 310002, China

${ }^{2}$ Department of Neurobiology, Key Laboratory of Medical Neurobiology of

the Ministry of Health of China, Zhejiang Province Key Laboratory of

Neurobiology, Zhejiang University School of Medicine, 866 Yuhangtang Rd,

Hangzhou, Zhejiang 310058, China

Full list of author information is available at the end of the article
}

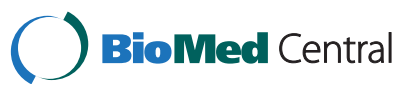

(c) 2015 Xu et al.; licensee BioMed Central. This is an Open Access article distributed under the terms of the Creative Commons Attribution License (http://creativecommons.org/licenses/by/4.0), which permits unrestricted use, distribution, and reproduction in any medium, provided the original work is properly credited. The Creative Commons Public Domain Dedication waiver (http://creativecommons.org/publicdomain/zero/1.0/) applies to the data made available in this article, unless otherwise stated. 


\section{Background}

Intracranial aneurysms (IAs) are a common type of cerebrovascular disorder with a high prevalence of $1-5 \%$ in the general population [1]. Although only $1-2 \%$ of IAs rupture annually, such a rupture can cause subarachnoid hemorrhage $(\mathrm{SAH})$, a devastating clinical consequence with high mortality (25-40\%) and morbidity ( $50 \%)$ constituting a major public health problem [2,3]. In contrast, IAs that are repaired before they rupture by microsurgical clipping or by endovascular coiling have a reported mortality as low as $0.6 \%$ and morbidity $<5 \%[4,5]$. Therefore, it would be advantageous to discover reliable predictive markers for screening individuals at risk for the development or rupture of an IA.

Recently, much work has focused on identifying susceptibility genes using genome-wide DNA linkage analyses or examining the single-nucleotide polymorphisms of individual genes that might be related to the development of IAs and their subsequent rupture [6-8]. Unfortunately, these studies have frequently yielded conflicting results and associations have not been replicated in other populations despite decades of research. These discrepancies may be due to genetic heterogeneity and a lack of knowledge of the molecular pathogenesis of IA formation, progression, and rupture. The molecular alterations within an aneurysm i.e. the dynamic molecular environment of the vasculature controlled by gene expression, may prove to be more predictive in distinguishing individuals at high risk of IA development or rupture. A series of human and animal studies of IA tissues using gene expression microarrays have highlighted the molecular changes resulting in endothelial dysfunction, the inflammatory response and degeneration of the vascular wall [9]. Although discovering the molecular markers in IA tissue has shed light on pathogenesis, it is neither practical nor feasible to use these molecules as biomarkers for screening individuals at risk for the development or IA rupture unless they can be confirmed in body fluids such as plasma and cerebrospinal fluid (CSF), which are easily accessible and ideal sources for biomarker discovery.

CSF is an important target for proteomic discovery of the disease-specific biomarkers of many brain disorders such as neurodegenerative diseases and traumatic brain injury $[10,11]$. Several markers have been identified in CSF that could predict susceptibility to aneurysmal SAH and post-SAH cerebral vasospasm [12-14]. However, the comprehensive characterization of CSF is still challenging because of its complexity and the high dynamic range of protein concentrations. To circumvent this difficulty, we focused on one subproteome of CSF: glycosylated proteins. Protein glycosylation is an abundant and biologically important post-translational modification. It is most commonly associated with membrane and secreted proteins. Indeed, many clinical markers and therapeutic targets such as Her2/neu (breast cancer) and prostate-specific antigen (prostate cancer) are glycoproteins $[15,16]$. In this study, we used a complementary proteomic approach which integrated the lectin-affinity column for glycoprotein enrichment and a multiplex quantitative proteomic method called iTRAQ (isobaric Tagging for Relative and Absolute protein Quantification), to simultaneously quantify relative changes in the glycoproteins of CSF obtained from patients with ruptured IAs (RIAs) and unruptured IAs (UIAs) compared to healthy controls (HCs) and disease controls (DCs). First, we identified a number of proteins that displayed quantitative changes unique to RIA, UIA or IA (discovery cohort, $n=40$ ). Then we confirmed the proteomic results in both CSF and plasma from a larger and independent cohort (validation cohort, $\mathrm{n}=80$ ). Finally, we tested the specificity and sensitivity of the candidate marker in CSF and plasma to evaluate whether it could be directly applied as a biomarker of IA rupture.

\section{Methods}

\section{Characterization of participants}

This study was approved by the Medical Ethics Committee of the Second Affiliated Hospital of Zhejiang University School of Medicine (Hangzhou, China), and written informed consent was given by all participants prior to the study. As a discovery step, we selected 40 age/gendermatched RIA, UIA, HC, and DC samples of CSF ( $\mathrm{n}=10$ for each group) from the patients consecutively admitted to the Department of Neurosurgery of the Second Affiliated Hospital from January 2008 to October 2010. We verified the proteomic results of the discovery set using an independent set of 80 age/gender-matched RIA, UIA, HC, and DC samples ( $\mathrm{n}=20$ for each group) from the patients consecutively admitted from January 2008 to October 2012. The demographics are listed in Table 1. The detailed clinical characteristics of all participants are listed in Additional file 1 of the supplementary materials. All study participants were Chinese of the Han ethnic group.

Both the discovery and validation samples followed the same inclusion and exclusion criteria. The HCs were selected among patients admitted for cranioplasty who had completely recovered from head trauma for at least six months. The HC inclusion criteria were as follows: 1) all the physical and neurological examination and laboratory tests were within the normal range; 2 ) no family history of IA and SAH in first-degree relatives; 3) confirmation that they did not harbor IA by digital subtraction angiography (DSA), three-dimensional CT angiography (CTA), or magnetic resonance angiography (MRA); 4) no heavy cigarette-smoking ( $<2$ packs/week) and alcohol use ( $<50 \mathrm{~g} /$ week). The DCs were selected among patients with brain tumors or other brain diseases and it was confirmed that they did not harbor IA by DSA, CTA, or MRA. The RIA group was selected among SAH patients with a 
Table 1 Demographics of patients

\begin{tabular}{llllll}
\hline \multicolumn{6}{l}{ Discovery cohort } \\
\hline Group & $\mathbf{N}$ & Gender (M:F) & $\boldsymbol{p}$ value & Age (mean \pm SD) & $\boldsymbol{p}$ value \\
\hline RIA & 10 & $8: 2$ & & $52.4 \pm 9.0$ & \\
UIA & 10 & $8: 2$ & & $55.2 \pm 3.9$ & \\
HC & 10 & $8: 2$ & & $52.8 \pm 6.9$ & \\
DC & 10 & $8: 2$ & $1^{\text {a }}$ & $54.6 \pm 4.2$ & $0.50^{\mathrm{b}}$ \\
\hline Validation cohort & & & \\
Group & $\mathbf{N}$ & M:F & $\boldsymbol{p}$ value & Age & \\
\hline RIA & 20 & $7: 13$ & & $51.7 \pm 8.4$ & \\
UIA & 20 & $7: 13$ & & $51.2 \pm 10.2$ & \\
HC & 20 & $7: 13$ & & $50.8 \pm 7.6$ & \\
DC & 20 & $7: 13$ & $1^{\text {a }}$ & $49.5 \pm 7.7$ & $0.85^{c}$ \\
\hline
\end{tabular}

${ }^{a}$ Gender difference between $d$ groups analyzed by the $x^{2}$ test. ${ }^{b}$ Age difference between groups in the discovery cohort analyzed by the nonparametric Kruskal-Wallis test (the variances were unequal by Bartlett's test, $p<0.05$ ). ${ }^{\mathrm{C}}$ Age difference between groups in the validation cohort analyzed by the Newman-Keuls multiple comparison test (the variances were equal by Bartlett's test, $p>0.05$ ).

diagnosis of IA using DSA. The UIA patients were found incidentally through CTA, MR imaging, or MRA performed for reasons other than suspicion of an index aneurysm and did not present any IA-related clinical symptoms or signs. The most common reasons leading to the discovery of an IA included nonspecific headaches or dizziness or regular physical examination. Most of the UIA patients (28 out of 30) were treated by coiling and clipping. Only 2 UIA patients harboring ophthalmic artery aneurysms underwent follow-up CTA examinations at intervals of 612 months.

\section{Collection of CSF and plasma}

Collection of CSF by lumbar puncture was performed as in our previous proteomic study [17]. All CSF samples were collected in the morning after overnight fasting. In addition, all CSF for proteomic analysis was taken from the 15th to 25th $\mathrm{ml}$ collected to limit variations arising from the rostral-caudal gradient, and $1.0 \mathrm{~mL}$ from each participant was used to generate a pooled sample, that is, a total of $10 \mathrm{ml}$ of CSF by pooling 10 participants for each group. Because the protein concentration in CSF is relatively low compared to plasma (CSF/plasma $1 / 100$ 200 ) and the protein profiles in CSF are similar to those in plasma, even a minor contamination of CSF with blood could significantly confound the interpretation of quantitative or qualitative proteomic analysis. Therefore, the CSF samples from patients who needed endovascular or neurosurgical treatment were collected before the operation. The samples from RIA and some DC patients with cerebral hemorrhage were collected one month after endovascular embolization or neurosurgical operation. The routine CSF biochemistry assays of those specimens (cell count, glucose, and total protein) were all in the normal range and there were $<10 \mathrm{RBCs}$ per $\mathrm{ml}$.

Blood samples $(10 \mathrm{ml})$ from the validation cohort were taken by venous puncture in conjunction with lumbar puncture, except for RIA and some DC patients with cerebral hemorrhage. The samples from those patients were collected within $2 \mathrm{~h}$ of admission to the hospital. Samples were collected in plastic tubes containing EDTA. Plasma was then separated by centrifugation at $5000 \mathrm{~g}$ at $4^{\circ} \mathrm{C}$ for $15 \mathrm{~min}$, and stored at $-80^{\circ} \mathrm{C}$ in aliquots of $0.1 \mathrm{ml}$ per tube until analysis.

\section{Isolation of glycoproteins from CSF using lectin affinity column}

Glycoproteins were isolated from aliquots of pooled CSF samples $(2 \mathrm{~mL})$ using a Qproteome Total Glycoprotein kit (Qiagen, Valencia, CA), which contains ConA and WGA lectins and can be used for a general enrichment of the total glycoprotein population from CSF. The sample was dried to $\sim 200 \mu \mathrm{L}$ with a SpeedVac (Thermo, Asheville, NC), then centrifuged at $1000 \mathrm{~g}$ for $5 \mathrm{~min}$, the supernatant transferred to another tube, and the pellets dissolved in $100 \mu \mathrm{L}$ of detergent supplied with the kit. The resultant samples were combined with the binding buffer along with protease inhibitor solution $(100 \times)$ before they were loaded onto the lectin column according to the manufacturer's instructions. Finally, the glycoproteins were eluted 6 times with $100 \mu \mathrm{L}$ of elution buffer with protease inhibitor solution. The pooled eluates were precipitated by adding 4 volumes of ice-cold acetone, incubated at $-20^{\circ} \mathrm{C}$ for $2 \mathrm{~h}$, and then centrifuged at $12,000 \mathrm{~g}$ in a pre-cool microcentrifuge at $4^{\circ} \mathrm{C}$ for $10 \mathrm{~min}$. The supernatant was removed and the air-dried pellet was re-suspended in $0.5 \mathrm{M}$ triethylammonium bicarbonate buffer. The protein concentration was determined by BCA assay (Thermo, Rockford, IL).

\section{iTRAQ labeling and two-dimensional liquid chromatography}

One hundred micrograms of protein from each group (RIA, UIA, HC, and DC) was digested with trypsin and then labeled with one of the four-iTRAQ ${ }^{\mathrm{mw}}$ reagents following the manufacturer's instructions (Applied Biosystems, Foster City, CA). Next, 4 samples labeled with iTRAQ reagents were combined (a total of $400 \mu \mathrm{g}$ protein), and separated into 6 fractions using a strong cation exchange (SCX) PolySulfoethyl A ${ }^{\mathrm{Tm}}$ column $(2.1 \times 200 \mathrm{~mm}, 5 \mu \mathrm{m}$, $300 \AA$ A, Poly LC, Columbia, MD).

The 6 SCX peptide fractions were dried down in a SpeedVac, dissolved in $0.5 \%$ trifluoroacetic acid, and separated by capillary $\mathrm{C} 18$ reverse phase chromatography (Dionex, Sunnyvale, CA). The eluted gradient was mixed with matrix solution and spotted onto a stainless-steel matrix-assisted laser desorption/ionization plate to form 
a $24 \times 24$ array for a total of 576 spots using the Probot ${ }^{\mathrm{Ts}}$ system (LC Packings, Sunnyvale, CA).

\section{Tandem MS analysis and protein identification and quantification}

Quantitative MS/MS analysis was carried out using a 4700 Proteomics Analyzer with TOF/TOF ${ }^{\mathrm{rm}}$ Optics (Applied Biosystems) in the reflector positive ion mode. The default calibration was performed before each run, and the mass accuracy calibrated to within $10 \mathrm{ppm}$ using calibration standards. The parameters were set as following: 800$4000 \mathrm{~m} / \mathrm{z}$ mass range with 1,000 shots per spectrum for MS analysis; a maximum of 15 peaks was selected per spot with a minimum signal-to-noise ratio of 50 and a cluster area of 500 for data-dependent MS/MS analysis. Proteins were identified using the Mascot (Matrix Science, Boston, MA) algorithm and searched against the International Protein Index (IPI; Version 3.10) human protein database from the European Bioinformatics Institute. For false discovery rate (FDR) analysis, the data were searched against the decoy database. A global FDR $<1 \%$ were included for protein identification. Proteins were quantified by averaging the iTRAQ ratios of all peptides identified. Individual quantification of the identified peptides was based on the individual ratios from signature ion-peak areas of the iTRAQ reagent tags of the identified peptides from RIA, UIA, and DC samples compared with the HC signature ion peak areas. Normalization, using a Gaussian distribution with a median of 1 when all peptides were considered between control and experimental groups, was performed after the iTRAQ ratios were calculated.

\section{Functional annotation of identified proteins}

The functions of the identified proteins were determined by searching against UniprotKB according to the IPI number. UniprotKB is a comprehensive, high-quality, protein database that contains a large amount of information on the biological functions of proteins derived from the research literature.

\section{Criteria for selecting candidate proteins for further validation}

Several criteria were used for validation: 1$)>50 \%$ increase or decrease ( iTRAQ ratio is $>1.5$ or $<0.67$; 2 ) markers annotated as glycoproteins with known glycosylation sites or probable/potential glycosylation sites in UniprotKB; 3) markers with a potential contribution to IA formation/ rupture; and 4) commercial antibodies available for Western blot or ELISA.

\section{Validation of Soluble AxI (sAxI) in CSF and plasma by ELISA}

Soluble (s) Axl in CSF and plasma were measured with an Axl Human ELISA kit (Abcam, Cambridge, MA) according to the manufacturer's instructions. Before measurement, CSF and plasma samples were diluted 1:100 and 1:1000 respectively with Assay Diluent A supplied with the kit. The absorbance at $450 \mathrm{~nm}$ was read with a microplate reader. The concentration of $\mathrm{sAXl}$ in CSF and plasma was determined using a standard curve.

\section{Statistical analysis}

Statistical analysis was performed with GraphPad Prism 5 (La Jolla, CA). The equality of variances was analyzed by Bartlett's test. One-way analysis of variance followed by the Newman-Keuls multiple comparison test was used for differences between groups when the variances were equal. Otherwise, the nonparametric Kruskal-Wallis test followed by Dunn's multiple comparison test was used. The correlation between CSF and plasma Axl levels was analyzed by Spearman's rank-based correlation coefficient. The specificity and sensitivity of the candidate marker were evaluated both graphically and statistically with receiver operating characteristic (ROC) curve methods. $P<0.05$ was considered statistically significant.

\section{Results}

\section{Identification of glycoproteins in human CSF}

By integrating the lectin-affinity purification of glycoproteins with a multiplex iTRAQ quantitative proteomic method, we were able to identify and quantify a total of 294 possible glycoproteins in human CSF with at least two peptides at $95 \%$ confidence (Additional file 2). Then we searched all of these proteins against UniprotKB according to the IPI number, and a unique UniprotKB accession number was assigned to each protein (listed in Additional file 3 of the Additional file materials). The functional classification of the identified 294 proteins is shown in Figure 1. This includes immune/inflammatory response, cell adhesion, extracellular matrix (ECM), protein synthesis or protein degradation, transport, apoptosis and anti-apoptosis, cell proliferation/differentiation/migration, signal transduction, metabolism, cell structure/cytoskeleton, and unknown functions. In comparison with UniprotKB, 142 of the 294 proteins were annotated as glycoproteins with known glycosylation sites or probable/potential glycosylation sites. Most of these glycoproteins are secreted or extracellular proteins (Additional file 4).

\section{Changed glycoproteins in CSF associated with RIA and UIA} In order to determine whether the changes in CSF glycoproteins were specific to IA or common in brain diseases, we had set up a group of DCs including diverse diseases such as brain tumors and cerebrovascular diseases other than aneurysms in addition to the HCs. Therefore, the proteins that were changed in the same direction in both IAs and DCs were excluded. For instance, extracellular superoxide dismutase [Cu-Zn] precursor (IPI00027827), 




Figure 1 Functional classification of 294 possible glycoproteins from human CSF. The proteins were classified into the following categories: immune/inflammatory response, cell adhesion, extracellular matrix (ECM), protein synthesis or protein degradation, transport, apoptosis and anti-apoptosis, cell proliferation/differentiation/ migration, signal transduction, metabolism, cell structure/cytoskeleton, and unknown functions. Proteins with multiple functions were assigned to the one that is best known.

not only displayed an increase in RIAs (iTRAQ ratio 1.60 \pm 0.17 ) but also in DCs (iTRAQ ratio $1.52 \pm 0.11$ ) compared to HCs, and would not be considered as a unique change in RIA. However, if a protein such as nectin-like protein 2 (IPI IPI00003813) displayed a significant decrease in RIAs (iTRAQ ratio $0.61 \pm 0.13$ ) but an increase in UIAs (iTRAQ ration $1.62 \pm 0.23$ ), its change would not only be considered unique to RIA but also to UIA. In addition, a protein would be considered a marker for IA if it displayed a significant change in the same direction in both RIA and UIA. With these criteria, we identified 40 proteins that displayed quantitative changes unique to RIA, 13 to UIA and 20 to IA (Table 2). These changed proteins were found to be involved in processes such as the immune/inflammatory response, cell adhesion, the ECM, protein synthesis and degradation, and apoptosis and anti-apoptosis, which are relevant to the pathogenesis of IA formation and rupture.

\section{Validation of sAxI as a potential marker for IA rupture}

Given that the current protein database is still incomplete, all the candidate protein markers identified and quantified by proteomics need to be validated before their application in clinical diagnosis is pursued extensively. Therefore we selected several proteins for further validation and Axl was the only one that was not only validated in the discovery CSF samples but also in the validation CSF/plasma samples. Axl, a tyrosine kinase receptor, which displayed a unique increase in the CSF of RIAs, was annotated as an N-linked glycoprotein in the database. A commercial human sAxl ELISA kit was used to validate the iTRAQ assay results.
We first confirmed the changes of sAxl in the pooled CSF samples that were used for proteomic discovery. The ELISA ratio of RIA/HC was $2.35 \pm 0.24$ while there was no significant difference between UIA versus. HC and DC versus. HC (Table 3), which was consistent with the iTRAQ results although the relative changes may vary between these two techniques simply because the dynamic range of iTRAQ and ELISA are different. Then we measured the sAxl concentration in the individual CSF samples of the discovery cohort. The results showed that the concentration of sAxl was increased in RIAs as compared to HCs $(p<0.01)$, DCs $(p<0.05)$, and UIAs $(p<0.01)$ (Figure 2A).

To determine the potential clinical utility of sAxl, we further assessed its levels in a separate larger cohort with paired CSF and plasma samples ( $\mathrm{n}=20$ per group). Compared with CSF, blood analysis has advantages as an approach to population-based disease screening because it is easier and less invasive. In this validation cohort, the mean sAxl of both CSF and plasma was significantly higher in RIAs (CSF, $0.143 \pm 0.055 \mathrm{nM}$; plasma, $1.670 \pm$ $0.802 \mathrm{nM}$ ) versus $\mathrm{HCs}$ (CSF, $0.095 \pm 0.057 \mathrm{nM}$; plasma, $1.073 \pm 0.460 \mathrm{nM}$ ) and UIAs (CSF, $0.070 \pm 0.040 \mathrm{nM}$; plasma, $1.115 \pm 0.659 \mathrm{nM}$ ) (Figures 2B and 3A) with levels roughly tenfold higher in plasma, although a wider range of values was found in the validation cohort than in a the discovery cohort, possibly reflecting the heterogeneity of clinical samples. Furthermore, Spearman's rank-based correlation coefficient analysis showed that CSF and plasma sAxl levels were strongly correlated $(\mathrm{r}=0.93,95 \%$ CI 0.87 $0.95, p<0.0001$; Figure 3B).

Finally, we used the ROC curve to evaluate the sensitivity and specificity of CSF and plasma sAxl in its ability to differentiate RIA from HC, DC, or UIA. The ROC analysis revealed that the CSF sAxl level performed well in discriminating RIA from UIA and $\mathrm{HC}$ with areas under the ROC curve (AUCs) of 0.89 (95\% CI 0.81-0.97, $p<0.0001$ ) and 0.83 (95\% CI 0.73-0.94, $p<0.0001$ ) (Figure 4A). The optimal CSF sAxl threshold of $0.12 \mathrm{nM}$ for discriminating RIA from UIA had a corresponding sensitivity/ specificity of $73.33 \% / 90 \%$ (Table 4). The plasma sAxl level also gave a significant result with AUCs of 0.72 (95\% CI $0.56-0.88, p=0.017$ ) and 0.71 (95\% CI 0.54-0.57, $p=0.027$ ) (Figure 4B). The optimal threshold for plasma sAxl was $1.7 \mathrm{nM}$ with corresponding sensitivity/specificity of 50\%/ $80 \%$ (Table 4).

\section{Discussion}

Extracellular proteins, including secreted, cell surface and transmembrane proteins are mostly glycosylated and likely to enter the body fluids to serve as potential biomarkers. Lectin-based approaches such as lectin affinity chromatography have been widely used in the discovery of glycoprotein biomarkers in diseases such as hepatocellular carcinoma [18], ovarian tumors [19] and prostate cancer 
Table 2 Protein changes unique to RIA, UIA, or IA

Protein name
Proteins unique to RIA
Immune/inflammatory response
Complement C1s subcomponent precursor
Pigment epithelium-derived factor precursor
Cell Adhesion

\section{Cell Adhesion}

Alcadein alpha-1

Calsyntenin-1 precursor

Contactin 2 precursor

Nectin-like protein 2

Neural cell adhesion molecule 1, $140 \mathrm{kDa}$ isoform precursor

\section{Extracellular Matrix}

Secretogranin III precursor

Splice isoform 1 of fibrinogen gamma chain precursor

Splice isoform 1 of fibulin-1 precursor

\section{Protein synthesis or protein degradation}

Hypothetical protein FLJ23322

Hypothetical protein FLJ23757

PREDICTED: KIAA1509

Splice isoform 1 of ecto-ADP-ribosyltransferase 3 precursor

TAR RNA loop binding protein

\section{Transport}

Afamin precursor

Ceruloplasmin precursor

Neuronal pentraxin I precursor

Serotransferrin precursor

Apoptosis or anti-apoptosis

\section{AXL receptor tyrosine kinase, isoform 1}

Splice isoform 1 of amine oxidase flavin containing domain protein 2

Beta-2-glycoprotein I precursor

Ephrin A1 isoform b precursor

Tyrosine phosphatase zeta polypeptide 2 HTPZP2

\section{Cell proliferation/differentiation/migration}

Splice isoform 1 of erythrocyte membrane protein band 4.2

\section{Signal transduction}

XPR1 protein

\section{Metabolism}

Guanylate binding protein 4

Splice isoform 2 of adenosine kinase

Splice isoform 2 of phospholipid transfer protein precursor

\section{Cell structure/cytoskeleton}

Nebulin

PREDICTED: dynein, cytoplasmic, heavy polypeptide 2

iTRAQ ratios $^{a}$

DC RIA UIA

$\begin{array}{llll}1.03 \pm 0.10 & \mathbf{1 . 2 8} \pm \mathbf{0 . 1 6} & 0.96 \pm 0.11 & 4 \\ 1.29 \pm 0.16 & \mathbf{0 . 7 6} \pm \mathbf{0 . 0 5} & 1.22 \pm 0.25 & 4\end{array}$

$\begin{array}{lll}1.22 \pm 0.17 & \mathbf{0 . 8 1} \pm \mathbf{0 . 0 3} & 1.32 \pm 0.11\end{array}$

$0.78 \pm 0.16 \quad \mathbf{1 . 2 0} \pm \mathbf{0 . 1 2} \quad 0.82 \pm 0.08$

$0.93 \pm 0.14 \quad \mathbf{1 . 2 0} \pm \mathbf{0 . 0 8} \quad 0.95 \pm 0.21 \quad 5$

$\begin{array}{llll}1.09 \pm 0.15 & \mathbf{0 . 6 1} \pm \mathbf{0 . 1 3} & 1.62 \pm 0.23 \quad 3\end{array}$

$0.88 \pm 0.12 \quad \mathbf{0 . 4 4} \pm \mathbf{0 . 1 4} \quad 0.97 \pm 0.21$

$\begin{array}{llll}0.83 \pm 0.05 & \mathbf{0 . 6 3} \pm \mathbf{0 . 0 4} & 1.00 \pm 0.16 & 3\end{array}$

$\begin{array}{lll}0.96 \pm 0.15 & \mathbf{1 . 5 2} \pm \mathbf{0 . 1 9} & 1.18 \pm 0.12\end{array}$

$\begin{array}{lll}1.03 \pm 0.12 & \mathbf{0 . 6 4} \pm \mathbf{0 . 1 1} & 1.09 \pm 0.15\end{array}$

$\begin{array}{lll}0.64 \pm 0.09 & \mathbf{1 . 4 3} \pm \mathbf{0 . 1 4} & 0.46 \pm 0.05 \quad 2\end{array}$

$\begin{array}{lll}1.07 \pm 0.08 & \mathbf{3 . 7 9} \pm \mathbf{0 . 5 3} & 0.99 \pm 0.10 \quad 2\end{array}$

$0.64 \pm 0.18 \quad \mathbf{1 . 4 3} \pm \mathbf{0 . 1 1} \quad 0.46 \pm 0.27 \quad 2$

$\begin{array}{lll}1.20 \pm 0.16 & \mathbf{3 . 0 5} \pm \mathbf{0 . 3 1} & 1.15 \pm 0.23 \quad 2\end{array}$

$0.71 \pm 0.25 \quad \mathbf{1 . 5 0} \pm \mathbf{0 . 1 6} \quad 1.14 \pm 0.08$

$\begin{array}{llll}1.18 \pm 0.08 & \mathbf{1 . 3 9} \pm \mathbf{0 . 0 5} & 1.19 \pm 0.12 & 4\end{array}$

$\begin{array}{llll}1.28 \pm 0.14 & \mathbf{0 . 7 6} \pm \mathbf{0 . 1 9} & 0.94 \pm 0.19 & 10\end{array}$

$\begin{array}{lll}1.07 \pm 0.10 & \mathbf{0 . 6 7} \pm \mathbf{0 . 0 4} & 0.88 \pm 0.03\end{array}$

$\begin{array}{llll}0.98 \pm 0.47 & \mathbf{0 . 7 6} \pm \mathbf{0 . 5 0} & 0.96 \pm 0.35 & 59\end{array}$

$\begin{array}{lll}1.12 \pm 0.11 & \mathbf{1 . 6 8} \pm \mathbf{0 . 0 7} & 0.87 \pm 0.16\end{array}$

$0.88 \pm 0.13 \quad \mathbf{0 . 3 8} \pm \mathbf{0 . 1 4} \quad 1.12 \pm 0.08 \quad 2$

$\begin{array}{llll}0.85 \pm 0.14 & \mathbf{1 . 4 8} \pm \mathbf{0 . 2 0} & 1.09 \pm 0.09\end{array}$

$0.75 \pm 0.06 \quad \mathbf{1 . 2 3} \pm \mathbf{0 . 0 3} \quad 1.18 \pm 0.02 \quad 2$

$\begin{array}{lll}1.32 \pm 0.21 & \mathbf{0 . 4 3} \pm \mathbf{0 . 1 2} & 1.20 \pm 0.19 \quad 2\end{array}$

$0.93 \pm 0.09 \quad 3.86 \pm \mathbf{0 . 4 9} \quad 1.53 \pm 0.11 \quad 2$

$\begin{array}{lll}0.88 \pm 0.18 & \mathbf{0 . 3 8} \pm \mathbf{0 . 0 7} & 1.12 \pm 0.15\end{array}$

$\begin{array}{lll}1.26 \pm 0.13 & \mathbf{0 . 6 8} \pm \mathbf{0 . 1 0} & 1.07 \pm 0.11 \quad 2\end{array}$

$0.88 \pm 0.08 \quad \mathbf{0 . 3 8} \pm \mathbf{0 . 1 5} \quad 1.12 \pm 0.11 \quad 2$

$\begin{array}{lll}0.71 \pm 0.23 & \mathbf{1 . 4 5} \pm \mathbf{0 . 0 7} & 0.86 \pm 0.16 \quad 2\end{array}$

$\begin{array}{llll}1.11 \pm 0.21 & \mathbf{1 . 7 4} \pm \mathbf{0 . 3 0} & 1.18 \pm 0.09 & 3\end{array}$

$\begin{array}{lll}0.88 \pm 0.09 & \mathbf{0 . 3 8} \pm \mathbf{0 . 1 2} & 1.12 \pm 0.16 \quad 2\end{array}$ 
Table 2 Protein changes unique to RIA, UIA, or IA (Continued)

\begin{tabular}{|c|c|c|c|c|}
\hline Splice isoform 3 o myosin Va & $0.68 \pm 0.29$ & $1.55 \pm 0.15$ & $1.14 \pm 0.08$ & 3 \\
\hline Titin & $1.08 \pm 0.14$ & $0.64 \pm 0.07$ & $1.07 \pm 0.13$ & 2 \\
\hline \multicolumn{5}{|l|}{ Unknown } \\
\hline 17-beta hydroxysteroid dehydrogenase & $0.80 \pm 0.21$ & $1.75 \pm 0.23$ & $1.12 \pm 0.16$ & 2 \\
\hline Chromosome 1 open reading frame 27 & $0.88 \pm 0.17$ & $0.38 \pm 0.12$ & $1.12 \pm 0.16$ & 2 \\
\hline JRK protein & $1.05 \pm 0.05$ & $1.77 \pm 0.17$ & $0.69 \pm 0.06$ & 2 \\
\hline PREDICTED: KIAA1522 protein & $0.60 \pm 0.16$ & $1.40 \pm 0.20$ & $0.84 \pm 0.12$ & 2 \\
\hline PREDICTED: similar to RIKEN CDNA 1700022 C21 & $0.80 \pm 0.21$ & $1.75 \pm 0.16$ & $1.12 \pm 0.10$ & 2 \\
\hline Taste receptor type 2 member 7 & $0.88 \pm 0.12$ & $0.38 \pm 0.06$ & $1.12 \pm 0.04$ & 2 \\
\hline Zinc finger MYND domain containing protein 19 & $0.97 \pm 0.03$ & $1.31 \pm 0.12$ & $0.86 \pm 0.08$ & 2 \\
\hline \multicolumn{5}{|l|}{ Proteins unique to UIA } \\
\hline \multicolumn{5}{|l|}{ Immune/inflammatory response } \\
\hline Complement C5 precursor & $1.34 \pm 0.15$ & $1.09 \pm 0.07$ & $0.68 \pm 0.05$ & 2 \\
\hline \multicolumn{5}{|l|}{ Cell Adhesion } \\
\hline Splice isoform 1 of neogenin precursor & $1.05 \pm 0.10$ & $1.10 \pm 0.14$ & $0.71 \pm 0.12$ & 3 \\
\hline \multicolumn{5}{|l|}{ Protein synthesis or protein degradation } \\
\hline Hypothetical protein FLJ34458 & $1.13 \pm 0.10$ & $0.94 \pm 0.12$ & $0.63 \pm 0.03$ & 2 \\
\hline SERPIND1 protein & $1.01 \pm 0.15$ & $1.00 \pm 0.08$ & $1.21 \pm 0.06$ & 4 \\
\hline \multicolumn{5}{|l|}{ Transport } \\
\hline Transthyretin precursor & $0.95 \pm 0.15$ & $0.88 \pm 0.07$ & $0.69 \pm 0.11$ & 5 \\
\hline \multicolumn{5}{|l|}{ Apoptosis or anti-apoptosis } \\
\hline Baculoviral IAP repeat-containing protein 4 & $1.20 \pm 0.11$ & $1.00 \pm 0.06$ & $0.47 \pm 0.08$ & 2 \\
\hline Nectin-like protein 2 & $1.09 \pm 0.15$ & $0.61 \pm 0.13$ & $1.62 \pm 0.23$ & 3 \\
\hline Nucleolysin TIAR & $0.93 \pm 0.07$ & $0.88 \pm 0.12$ & $0.68 \pm 0.06$ & 3 \\
\hline Splice isoform 2 of signal transducer and activator of transcription 1-alpha/beta & $1.13 \pm 0.06$ & $0.94 \pm 0.03$ & $0.63 \pm 0.10$ & 2 \\
\hline \multicolumn{5}{|l|}{ Cell proliferation/differentiation/migration } \\
\hline Condensin subunit 2 & $0.85 \pm 0.10$ & $0.87 \pm 0.09$ & $0.55 \pm 0.08$ & 3 \\
\hline \multicolumn{5}{|l|}{ Signal transduction } \\
\hline Grb10 interacting GYF protein 1 & $1.13 \pm 0.05$ & $0.94 \pm 0.14$ & $0.63 \pm 0.02$ & 2 \\
\hline \multicolumn{5}{|l|}{ Metabolism } \\
\hline Vitamin D-binding protein precursor & $1.14 \pm 0.10$ & $1.06 \pm 0.18$ & $1.29 \pm 0.09$ & 6 \\
\hline \multicolumn{5}{|l|}{ Unknown } \\
\hline JRK protein & $1.05 \pm 0.05$ & $1.77 \pm 0.17$ & $0.69 \pm 0.06$ & 2 \\
\hline \multicolumn{5}{|l|}{ Proteins changed both in RIA and UIA } \\
\hline \multicolumn{5}{|l|}{ Immune/inflammatory response } \\
\hline Complement C3 precursor & $0.91 \pm 0.40$ & $1.28 \pm 0.42$ & $1.25 \pm 0.33$ & 16 \\
\hline Splice isoform 2 of interleukin-17E precursor & $1.21 \pm 0.09$ & $0.62 \pm 0.13$ & $0.67 \pm 0.12$ & 2 \\
\hline \multicolumn{5}{|l|}{ Cell Adhesion } \\
\hline Neurocan core protein precursor & $0.91 \pm 0.15$ & $0.44 \pm 0.10$ & $0.54 \pm 0.12$ & 3 \\
\hline Splice isoform 2 of fibrinogen alpha/alpha-E chain precursor & $1.14 \pm 0.16$ & $0.56 \pm 0.12$ & $0.78 \pm 0.13$ & 4 \\
\hline \multicolumn{5}{|l|}{ Extracellular Matrix } \\
\hline Extracellular matrix protein 1 & $1.16 \pm 0.12$ & $0.55 \pm 0.15$ & $0.69 \pm 0.13$ & 3 \\
\hline Metalloproteinase inhibitor 1 precursor & $1.07 \pm 0.11$ & $1.25 \pm 0.08$ & $1.31 \pm 0.12$ & 4 \\
\hline
\end{tabular}


Table 2 Protein changes unique to RIA, UIA, or IA (Continued)

\begin{tabular}{|c|c|c|c|c|}
\hline \multicolumn{5}{|l|}{ Protein synthesis or protein degradation } \\
\hline Acheron, isoform 1 & $0.97 \pm 0.03$ & $1.31 \pm 0.08$ & $1.64 \pm 0.16$ & 2 \\
\hline \multicolumn{5}{|l|}{ Transport } \\
\hline Apolipoprotein D precursor & $1.19 \pm 0.08$ & $1.43 \pm 0.12$ & $1.47 \pm 0.11$ & 3 \\
\hline Serum albumin precursor & $1.08 \pm 0.17$ & $1.39 \pm 0.11$ & $1.59 \pm 0.12$ & 4 \\
\hline \multicolumn{5}{|l|}{ Cell proliferation/differentiation/migration } \\
\hline Septin 10 isoform 2 & $1.08 \pm 0.02$ & $1.28 \pm 0.05$ & $1.46 \pm 0.08$ & 2 \\
\hline \multicolumn{5}{|l|}{ Signal transduction } \\
\hline Adrenomedullin 2 precursor & $1.08 \pm 0.04$ & $1.30 \pm 0.07$ & $1.28 \pm 0.04$ & 2 \\
\hline CD59 glycoprotein precursor & $0.90 \pm 0.07$ & $0.49 \pm 0.03$ & $0.64 \pm 0.06$ & 2 \\
\hline Neuronal pentraxin receptor isoform 1 & $1.00 \pm 0.02$ & $0.74 \pm 0.05$ & $0.50 \pm 0.11$ & 3 \\
\hline \multicolumn{5}{|l|}{ Metabolism } \\
\hline Lumican precursor & $0.99 \pm 0.13$ & $1.57 \pm 0.04$ & $1.36 \pm 0.13$ & 3 \\
\hline $\mathrm{N}$-acetylgalactosamine-4-O-sulfotransferase & $0.88 \pm 0.05$ & $1.21 \pm 0.08$ & $1.28 \pm 0.07$ & 2 \\
\hline \multicolumn{5}{|l|}{ Cell structure/cytoskeleton } \\
\hline Keratin 1 & $1.49 \pm 0.17$ & $0.66 \pm 0.07$ & $0.82 \pm 0.07$ & 3 \\
\hline PREDICTED: similar to keratin, type I cytoskeletal 18 (cytokeratin 18) (K18) (CK 18) & $1.73 \pm 0.28$ & $0.69 \pm 0.06$ & $0.76 \pm 0.08$ & 2 \\
\hline \multicolumn{5}{|l|}{ Unknown } \\
\hline DJ570F3.6 & $0.69 \pm 0.23$ & $2.36 \pm 0.36$ & $2.14 \pm 0.24$ & 2 \\
\hline MUF1 protein & $1.09 \pm 0.08$ & $0.72 \pm 0.10$ & $0.72 \pm 0.11$ & 3 \\
\hline PREDICTED: hypothetical protein XP_291007 & $0.97 \pm 0.06$ & $0.59 \pm 0.04$ & $0.60 \pm 0.04$ & 2 \\
\hline
\end{tabular}

a $\mathrm{iTRAQ}$ ratios shown as mean \pm S.D. obtained by averaging the iTRAQ ratios of individual peptides. The differences between groups were analyzed by one-way ANOVA followed by Newman-Keuls multiple comparison test. ${ }^{b}$ Numbers of identified peptides used for calculating the iTRAQ ratios. The iTRAQ ratio in bold showed significant differences compared to the other group(s) $(p<0.05)$.

[20]. In the present study, we applied lectin-affinity enrichment of glycoproteins coupled with quantitative iTRAQlabeling proteomic techniques to discover candidate biomarkers for predicting the risk of development or rupture of IA. Using this quantitative glycoproteomics, we identified CSF proteins either distinctively changed in RIA or UIA or commonly changed in RIA and UIA. The former could be used as candidate biomarkers to predict the risk of IA rupture, while the latter could be used to distinguish individuals at risk of IA formation.

A total of 73 CSF proteins that were differentially expressed in IA compared to controls were identified.

Table 3 Comparison of iTRAQ ratio versus ELISA ratio for sAxl in pooled CSF samples

\begin{tabular}{|c|c|c|c|}
\hline Group & $\begin{array}{l}\text { iTRAQ ratio } \\
\text { (mean } \pm \text { SD) }\end{array}$ & $\begin{array}{l}\text { ELISA ratio }^{a} \\
(\text { mean } \pm S D)\end{array}$ & $\begin{array}{l}\text { ELISA concentration } \\
(\mathrm{nM})(\text { mean } \pm \text { SD) }\end{array}$ \\
\hline $\mathrm{RIA}$ & $1.68 \pm 0.07$ & $2.35 \pm 0.24^{*}, \#, \$$ & $0.204 \pm 0.017^{*}, \#, \$$ \\
\hline UIA & $0.87 \pm 0.16$ & $0.98 \pm 0.47$ & $0.082 \pm 0.030$ \\
\hline $\mathrm{DC}$ & $1.12 \pm 0.11$ & $1.52 \pm 0.48$ & $0.128 \pm 0.022$ \\
\hline $\mathrm{HC}$ & - & - & $0.088 \pm 0.017$ \\
\hline
\end{tabular}

${ }^{a}$ ELISA ratio obtained from 3 independent measurements of sAxl concentration in the RIA, UIA, and DC groups divided by the HC group. The differences between groups were analyzed by one -way ANOVA followed by the Newman-Keuls multiple comparison test. ${ }^{*} p<0.01$ for RIA vs UIA; ${ }^{p} p<0.01$ for RIA vs HC; ${ }^{\$} p<0.05$ for RIA vs DC.
These candidate markers fall into all 11 functional categories: 1) immune/inflammatory response, 2) cell adhesion, 3) ECM, 4) protein synthesis or protein degradation, 5) transport, 6) apoptosis and anti-apoptosis, 7) cell proliferation/differentiation/migration, 8) signal transduction, 9) metabolism, 10) cell structure/cytoskeleton, and 11) unknown functions. Among these proteins, some have been implicated in the pathogenesis of IA in previous studies, such as the complement components C1, C3 and C5 [21,22] and the ECM remodeling-related proteins ECM1 and TIMP1 [23]. Furthermore, we also found a number of proteins that have never been reported but are involved in the injury and/or repair of vascular epithelial cells, cell adhesion and migration, and angiogenesis, such as pigment epithelium-derived factor (PEDF) [24], nectinlike protein 2 (NECL2) [25], ephrin A1 [26], neogenin [27], acheron [28], lumican [29] and Axl [30-33].

Of these novel potential biomarkers, receptor protein kinase Axl was distinctively increased in RIA and its differential expression was confirmed in an independent cohort not only in CSF but also in the paired plasma. Axl, which was originally cloned from cancer cells, is broadly expressed in a variety of cells including vascular epithelial cells, smooth muscle cells and fibroblasts [32,33]. Overexpression and an increase of Axl activity have been found 

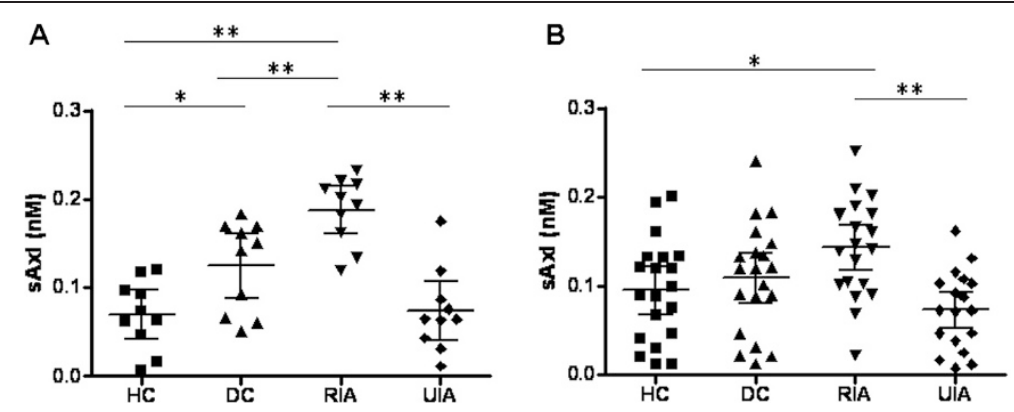

Figure 2 Confirmation of the changes of CSF sAxl levels in both discovery and validation cohorts using ELISA. (A) SAxI concentration in individual CSF samples from the discovery cohort ( $n=10$ per group). (B) $s A x \mid$ concentration in individual CSF samples from the validation cohort ( $n=20$ per group). ${ }^{*} p<0.05$ and ${ }^{*} p<0.01$ (Newman-Keuls test). Error bars represent the $95 \%$ confidence interval of the mean.

in cancer, chronic immune disorders and cardiovascular diseases [30,32,34,35]. Axl-dependent signaling regulates various functions, including survival, growth, aggregation, migration and anti-inflammation, in multiple cell-types. The mechanisms of Axl receptor activation/inactivation are not completely understood. Typically, growth-arrestspecific protein 6 (Gas6), the ligand with the highest affinity for Axl, binds to Axl receptor to form an initial 1:1 Gas6-Axl complex followed by the dimerization of two 1:1 Gas6-Axl complexes, which is a ligand-dependent activation that usually occurs under physiological conditions [32]. During pathophysiological conditions with increased oxidative stress and experimental receptor overexpression, Axl can be activated and autophosphorylated via ligandindependent homophilic dimerization [36]. Furthermore, release of a soluble form of Axl ( $\mathrm{sAxl}$ ), the extracellular domain of Axl, is another important feature of Axl receptor biology. A specific proteinase that mediates the cleavage of sAxl has yet to be identified. Axl signaling has mainly been implicated in cancer. However, a rapid increase in publications supports the importance of Axl in other chronic pathological conditions [34,35]. A correlation of plasma concentrations of Gas6 and sAxl with disease activity in systemic lupus erythematosus has recently been reported [31]. Ekam et al. found that plasma Gas6 correlated positively and sAxl correlates negatively with abdominal aortic aneurysms (AAA) size and the Gas6/sAxl ratio may be useful as an AAA biomarker [37]. Nevertheless, more studies are required to determine the role of Axl in the pathogenesis of IA formation, progression, and rupture, and Axl may be a promising biomarker for predicting the risk of IA rupture upon further validation in larger cohorts.

There are some potential limitations in our study. First, we used patients who had completely recovered from head trauma for at least six months as HCs. They may not be considered as "really healthy" controls even if all the results of the physical and neurological examinations and laboratory tests were within the normal range. The longest time period reported in previous studies to analyze inflammatory factors or cytokines in CSF was 22 days and they had returned the normal range by this time [38]. Most of the control patients we recruited had recovered from head trauma for about one year, in order to reduce the effect of disease as much as possible. Second, the CSF samples from the RIA group were collected one month after endovascular embolization or a neurosurgical operation, since contamination of CSF with blood could significantly confound the interpretation of quantitative or qualitative proteomic analysis of CSF. This might not be the ideal
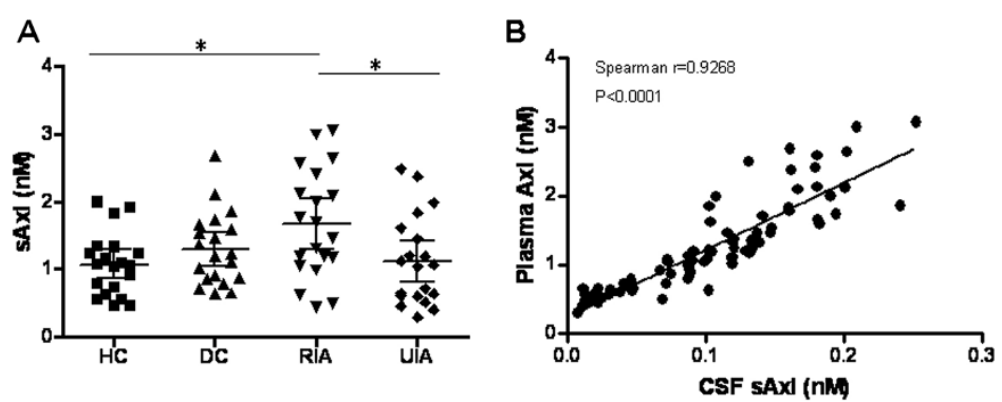

Figure $3 \mathrm{sAxl}$ concentration in plasma samples from the validation cohort (A) and its correlation with CSF samples (B). The error bars represent the $95 \%$ confidence interval of the mean. The Spearman correlation was significant between plasma and CSF sAxI concentration $(r=0.9268 ; 95 \% \mathrm{Cl}, 0.8865-09532, \mathrm{p}<0.0001)$. 


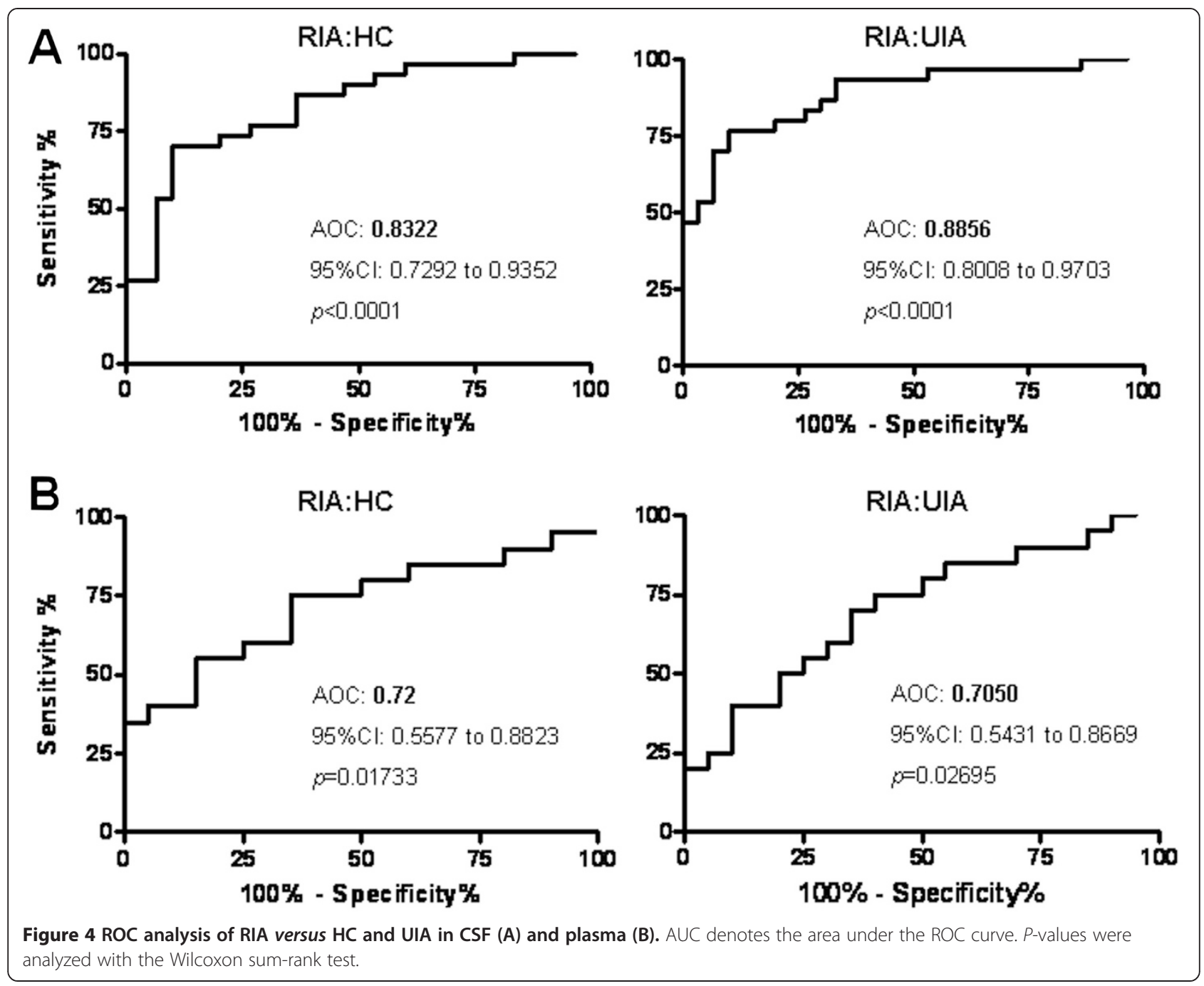

time point to collect CSF for RIA. However, considering the inability to collect RIA CSF samples before rupture, it is still reasonable at the discovery step. Moreover, the blood samples of RIA were taken within $2 \mathrm{~h}$ after the patients were admitted to hospital. Our results demonstrated that the sAxl level was not only elevated in CSF but also in plasma, which excluded the chance that the increase of sAxl was a result of a restorative process after aneurysm rupture. Third, since most of our UIA patients were treated with coiling or clipping, it is possible that some of them had a risk of rupture. It is interesting that two follow-up UIA patients with ophthalmic artery aneurysms had a relatively low concentration of CSF sAxl $(0.076 \mathrm{nM}$ and $0.043 \mathrm{nM}$ ). Therefore, next step we will measure the dynamic sAxl levels in CSF and plasma from patients harboring incidental IA and follow-up with CTA every 6 months looking for a correlation between the sAxl levels and the anatomical and/or morphological changes in the aneurysm as signs of impending rupture. So far, we have not recruited enough patients to draw conclusions. Finally,
Table 4 Sensitivity and specificity of sAxI in CSF and plasma to discriminate between RIA and UIA

\begin{tabular}{|c|c|c|c|c|}
\hline \multicolumn{5}{|c|}{ sAxl in CSF ( $n=30 /$ group) } \\
\hline $\begin{array}{l}\text { Cut off } \\
\text { (nM) }\end{array}$ & $\begin{array}{l}\text { Sensitivity \% } \\
(95 \% \mathrm{Cl})\end{array}$ & $\begin{array}{l}\text { Specificity \% } \\
(95 \% \text { Cl) }\end{array}$ & LR+ & LR- \\
\hline 0.02 & 100.00 (88.43 to 100.00$)$ & 13.33 (3.76 to 30.72 ) & 1.15 & 0.00 \\
\hline 0.09 & 90.00 (73.47 to 97.89$)$ & 66.67 (47.19 to 82.71$)$ & 2.70 & 0.15 \\
\hline 0.10 & 86.67 (69.28 to 96.24$)$ & 70.00 (50.60 to 85.27$)$ & 2.89 & 0.19 \\
\hline 0.11 & 76.67 (57.72 to 90.07$)$ & 83.33 (65.28 to 94.36$)$ & 4.60 & 0.28 \\
\hline 0.12 & 73.33 (54.11 to 87.72$)$ & 90.00 (54.11 to 87.72$)$ & 7.33 & 0.30 \\
\hline 0.14 & 63.33 (43.86 to 80.07 ) & 93.33 (77.93 to 99.18$)$ & 9.50 & 0.39 \\
\hline \multicolumn{5}{|c|}{ sAxl in plasma ( $n=20 /$ group) } \\
\hline $\begin{array}{l}\text { Cut off } \\
(\mathrm{nM})\end{array}$ & $\begin{array}{l}\text { Sensitivity \% } \\
(95 \% \mathrm{Cl})\end{array}$ & $\begin{array}{l}\text { Specificity \% } \\
(95 \% \mathrm{Cl})\end{array}$ & LR+ & LR- \\
\hline$\overline{0.4}$ & $100(83.16$ to 100.0$)$ & $5(0.1265$ to 24.87$)$ & 1.05 & 0.00 \\
\hline 0.5 & 90 (68.30 to 98.77$)$ & 15 (3.207 to 37.89$)$ & 1.06 & 0.67 \\
\hline 1 & 80 (56.34 to 94.27$)$ & 45 (23.06 to 68.47$)$ & 1.45 & 0.44 \\
\hline 1.2 & 65 (40.78 to 84.61$)$ & 65 (40.78 to 84.61$)$ & 1.86 & 0.54 \\
\hline 1.7 & 50 (27.20 to 72.80$)$ & 80 (56.34 to 94.27$)$ & 2.50 & 0.63 \\
\hline
\end{tabular}

95\% Cl, 95\% confidence interval; LR+, positive likelihood ratio; LR-, negative likelihood ratio. 
the sensitivity and specificity of plasma sAxl is probably not sufficient to identify RIA patients although the overall expression levels of sAxl from RIA were significantly higher than UIAs and controls. On the one hand, a larger population is needed for further validation. On the other hand, more candidate biomarkers need to be validated.

\section{Conclusions}

In summary, we successfully used lectin-affinity purification and iTRAQ quantitative proteomics approaches to identify candidate biomarkers for predicting the risk of development or rupture of IAs. One candidate biomarker, sAxl, was confirmed to be elevated both in CSF and plasma of RIA patients. The sAxl levels in CSF discriminated RIA from UIA with relatively high sensitivity and specificity, indicating that sAxl might serve as a promising biomarker to predict the rupture of IA.

\section{Additional files}



\section{Abbreviations}

AAA: Abdominal aortic aneurysms; AUC: The area under ROC curve; CSF: Cerebrospinal fluid; CTA: CT angiography; DC: Disease control; DSA: Digital subtraction angiography; ECM: Extracellular matrix; ELISA: Enzyme-linked immunosorbent assay; Gas6: Growth-arrest-specific protein 6; HC: Healthy control; IA: Intracranial aneurysms; IPI: International Protein Index; ITRAQ: Isobaric Tagging for Relative and Absolute protein Quantification; MRA: Magnetic resonance angiography; MS: Mass spectrometry; RIA: Ruptured intracranial aneurysm; ROC: Receiver operating characteristic; SAH: Subarachnoid hemorrhage; SCX: Strong cation exchange; UIA: Unruptured intracranial aneurysm.

\section{Competing interests}

The authors declare that they have no competing interests.

\section{Authors' contributions}

JX contributed to the study design, coordinating the clinical sample collection, data analysis, literature research, and manuscript drafting. FM and WY participated in the clinical sample collection, sample preparation, and MS data analysis. SQ and SX carried out the ELISA assays, and statistical analysis. YL was involved in the sample preparation and MS data analysis. JL participated in the design of the study and helped to draft the manuscript. JZ conceived of the study and participated in its design and coordination. JJ was involved in the study design, sample preparation, data analysis, literature research, and manuscript drafting. All authors read and approved the final manuscript.

\section{Acknowledgements}

The authors thank Professor Yimin Zhu for consulting with the statistical analysis and Professor lain C. Bruce and Mr. Mauricio Pena for help in editing the manuscript. This research was supported by the National Nature Science Foundation of China (81171199 to J. Jin) and the Talents Project of Health Department of Zhejiang Province (2011RCB022 to J. Xu).

\section{Author details}

'Department of Neurosurgery, The Second Affiliated Hospital of Zhejiang University School of Medicine, Hangzhou, Zhejiang 310002, China. ${ }^{2}$ Department of Neurobiology, Key Laboratory of Medical Neurobiology of the Ministry of Health of China, Zhejiang Province Key Laboratory of
Neurobiology, Zhejiang University School of Medicine, 866 Yuhangtang Rd, Hangzhou, Zhejiang 310058, China. ${ }^{3}$ Department of Joint Surgery, Shandong Provincial Hospital Affiliated with Shandong University, Jinan, Shandong 250021, China.

Received: 10 June 2014 Accepted: 20 February 2015

Published online: 05 March 2015

\section{References}

1. Huang MC, Baaj AA, Downes $K$, Youssef AS, Sauvageau E, van Loveren HR, et al. Paradoxical trends in the management of unruptured cerebral aneurysms in the United States: analysis of nationwide database over a 10-year period. Stroke. 2011;42(6):1730-5.

2. Nieuwkamp DJ, Setz LE, Algra A, Linn FH, de Rooij NK, Rinkel GJ. Changes in case fatality of aneurysmal subarachnoid haemorrhage over time, according to age, sex, and region: a meta-analysis. Lancet Neurol. 2009;8(7):635-42.

3. Lovelock CE, Rinkel GJ, Rothwell PM. Time trends in outcome of subarachnoid hemorrhage: Population-based study and systematic review. Neurology. 2010;74(19):1494-501.

4. Brinjikji W, Rabinstein AA, Nasr DM, Lanzino G, Kallmes DF, Cloft HJ. Better outcomes with treatment by coiling relative to clipping of unruptured intracranial aneurysms in the United States, 2001-2008. AJNR Am J Neuroradiol. 2011;32(6):1071-5.

5. Steiner T, Juvela S, Unterberg A, Jung C, Forsting M, Rinkel G. European Stroke Organization guidelines for the management of intracranial aneurysms and subarachnoid haemorrhage. Cerebrovasc Dis. 2013;35(2):93-112.

6. Marchese E, Vignati A, Albanese A, Nucci CG, Sabatino G, Tirpakova B, et al. Comparative evaluation of genome-wide gene expression profiles in ruptured and unruptured human intracranial aneurysms. J Biol Regul Homeost Agents. 2010;24(2):185-95.

7. Weinsheimer S, Goddard KA, Parrado AR, Lu Q, Sinha M, Lebedeva ER, et al. Association of kallikrein gene polymorphisms with intracranial aneurysms. Stroke. 2007;38(10):2670-6.

8. Baumann A, Devaux Y, Audibert G, Zhang L, Bracard S, Colnat-Coulbois S, et al. Gene expression profile of blood cells for the prediction of delayed cerebral ischemia after intracranial aneurysm rupture: a pilot study in humans. Cerebrovasc Dis. 2013;36(3):236-42.

9. Pera J, Korostynski M, Krzyszkowski T, Czopek J, Slowik A, Dziedzic T, et al. Gene expression profiles in human ruptured and unruptured intracranial aneurysms: what is the role of inflammation? Stroke. 2010;41(2):224-31.

10. Abdi F, Quinn JF, Jankovic J, McIntosh M, Leverenz JB, Peskind E, et al. Detection of biomarkers with a multiplex quantitative proteomic platform in cerebrospinal fluid of patients with neurodegenerative disorders. J Alzheimers Dis. 2006;9(3):293-348.

11. Ahmed F, Gyorgy A, Kamnaksh A, Ling G, Tong L, Parks S, et al. Time-dependent changes of protein biomarker levels in the cerebrospinal fluid after blast traumatic brain injury. Electrophoresis. 2012;33(24):3705-11.

12. Schebesch KM, Brawanski A, Bele S, Schodel P, Herbst A, Brundl E, et al. Neuropeptide $Y$ ? an early biomarker for cerebral vasospasm after aneurismal subarachnoid hemorrhage. Neurol Res 2013, [Epub ahead of print].

13. Suzuki H, Kanamaru K, Shiba M, Fujimoto M, Imanaka-Yoshida K, Yoshida T, et al. Cerebrospinal fluid tenascin-C in cerebral vasospasm after aneurysmal subarachnoid hemorrhage. J Neurosurg Anesthesiol. 2011;23(4):310-7.

14. Moussouttas M, Huynh TT, Khoury J, Lai EW, Dombrowski K, Pello S, et al. Cerebrospinal fluid catecholamine levels as predictors of outcome in subarachnoid hemorrhage. Cerebrovasc Dis. 2012;33(2):173-81.

15. Roth J. Protein $\mathrm{N}$-glycosylation along the secretory pathway: relationship to organelle topography and function, protein quality control, and cell interactions. Chem Rev. 2002;102(2):285-303.

16. Shepard HM, Lewis GD, Sarup JC, Fendly BM, Maneval D, Mordenti J, et al. Monoclonal antibody therapy of human cancer: taking the HER2 protooncogene to the clinic. J Clin Immunol. 1991;11(3):117-27.

17. Xu J, Chen J, Peskind ER, Jin J, Eng J, Pan C, et al. Characterization of proteome of human cerebrospinal fluid. Int Rev Neurobiol. 2006;73:29-98.

18. Na K, Lee EY, Lee HJ, Kim KY, Lee H, Jeong SK, et al. Human plasma carboxylesterase 1, a novel serologic biomarker candidate for hepatocellular carcinoma. Proteomics. 2009;9(16):3989-99.

19. Kuzmanov U, Smith CR, Batruch I, Soosaipillai A, Diamandis A, Diamandis EP. Separation of kallikrein 6 glycoprotein subpopulations in biological fluids by anion-exchange chromatography coupled to ELISA and identification by mass spectrometry. Proteomics. 2012;12(6):799-809. 
20. Jankovic MM, Kosanovic MM. Glycosylation of urinary prostate-specific antigen in benign hyperplasia and cancer: assessment by lectin-binding patterns. Clin Biochem. 2005;38(1):58-65

21. Tulamo R, Frosen J, Junnikkala S, Paetau A, Kangasniemi M, Pelaez J, et al. Complement system becomes activated by the classical pathway in intracranial aneurysm walls. Lab Invest. 2009;90(2):168-79.

22. Tulamo R, Frosen J, Junnikkala S, Paetau A, Pitkaniemi J, Kangasniemi M, et al. Complement activation associates with saccular cerebral artery aneurysm wall degeneration and rupture. Neurosurgery. 2006;59(5):1069-76. discussion 1076-1067.

23. Aoki T, Kataoka H, Moriwaki T, Nozaki K, Hashimoto N. Role of TIMP-1 and TIMP-2 in the progression of cerebral aneurysms. Stroke. 2007;38(8):2337-45.

24. Becerra SP, Notario V. The effects of PEDF on cancer biology: mechanisms of action and therapeutic potential. Nat Rev Cancer. 2013;13(4):258-71.

25. Fournier G, Garrido-Urbani S, Reymond N, Lopez M. Nectin and nectin-like molecules as markers, actors and targets in cancer. Med Sci (Paris). 2010;26(3):273-9.

26. Coulthard MG, Morgan M, Woodruff TM, Arumugam TV, Taylor SM, Carpenter TC, et al. Eph/Ephrin signaling in injury and inflammation. Am J Pathol. 2012;181(5):1493-503.

27. Enns CA, Ahmed R, Zhang AS. Neogenin interacts with matriptase-2 to facilitate hemojuvelin cleavage. J Biol Chem. 2012;287(42):35104-17.

28. Sun R, Chen W, Zhao X, Li T, Song Q. Acheron regulates vascular endothelial proliferation and angiogenesis together with Id 1 during wound healing. Cell Biochem Funct. 2011;29(8):636-40.

29. Malinowski M, Pietraszek K, Perreau C, Boguslawski M, Decot V, Stoltz JF, et al. Effect of lumican on the migration of human mesenchymal stem cells and endothelial progenitor cells: involvement of matrix metalloproteinase-14. PLoS One. 2012;7(12):e50709.

30. Ammoun S, Provenzano L, Zhou L, Barczyk M, Evans K, Hilton DA, et al. Axl/Gas6/NFkappaB signalling in schwannoma pathological proliferation, adhesion and survival. Oncogene. 2014;33(3):336-46.

31. Ekman C, Jonsen A, Sturfelt G, Bengtsson AA, Dahlback B. Plasma concentrations of Gas6 and sAxl correlate with disease activity in systemic lupus erythematosus. Rheumatology (Oxford). 2011;50(6):1064-9.

32. Korshunov VA. Axl-dependent signalling: a clinical update. Clin Sci (Lond). 2012;122(8):361-8.

33. O'Bryan JP, Frye RA, Cogswell PC, Neubauer A, Kitch B, Prokop C, et al. axl, a transforming gene isolated from primary human myeloid leukemia cells, encodes a novel receptor tyrosine kinase. Mol Cell Biol. 1991;11(10):5016-31.

34. Gallicchio M, Mitola S, Valdembri D, Fantozzi R, Varnum B, Avanzi GC, et al. Inhibition of vascular endothelial growth factor receptor 2-mediated endothelial cell activation by Axl tyrosine kinase receptor. Blood. 2005:105(5):1970-6.

35. Lemke G, Rothlin CV. Immunobiology of the TAM receptors. Nat Rev Immunol. 2008:8(5):327-36.

36. Smolock EM, Korshunov VA. Pharmacological inhibition of Axl affects smooth muscle cell functions under oxidative stress. Vascul Pharmacol. 2010;53(3-4):185-92.

37. Ekman C, Site DF, Gottsater A, Lindblad B, Dahlback B. Plasma concentrations of growth arrest specific protein 6 and the soluble form of its tyrosine kinase receptor Axl as markers of large abdominal aortic aneurysms. Clin Biochem. 2010;43(1-2):110-4

38. Csuka E, Morganti-Kossmann MC, Lenzlinger PM, Joller H, Trentz O, Kossmann T. IL-10 levels in cerebrospinal fluid and serum of patients with severe traumatic brain injury: relationship to IL-6, TNF-alpha, TGF-beta1 and blood-brain barrier function. J Neuroimmunol. 1999;101(2):211-21.

\section{Submit your next manuscript to BioMed Central and take full advantage of:}

- Convenient online submission

- Thorough peer review

- No space constraints or color figure charges

- Immediate publication on acceptance

- Inclusion in PubMed, CAS, Scopus and Google Scholar

- Research which is freely available for redistribution

Submit your manuscript at www.biomedcentral.com/submit 